\title{
Bibliotecas Nacionais e aplicativos móveis: análise de serviços on-line disponíveis em aplicativos para Android
}

\author{
Enrique Muriel-Torrado \\ Universidade Federal de Santa Catarina, Pós-Graduação em Ciência da Informação (PGCIN), \\ Florianópolis, SC, Brasil \\ enrique.muriel@ufsc.br
}

\begin{abstract}
Amanda Soares
Universidade Federal de Santa Catarina, Departamento de Engenharia do Conhecimento, Florianópolis,

SC, Brasil

amandasoaresbiblio@gmail.com
\end{abstract}

DOI: https://doi.org/10.265/rici.v13.n3.25276

Recebido/Recibido/Received: 2019-06-24

Aceitado/Aceptado/Accepted: 2020-06-22

Resumo: $\mathrm{O}$ objetivo do presente estudo foi analisar os serviços online disponíveis em aplicativos para Android das Bibliotecas Nacionais (BN) dos países do Índice de Desenvolvimento de Tecnologia da Informação e Comunicação (IDI) que possuem dentre suas línguas oficiais o espanhol, inglês ou português, resultando no total de 83 países. Inicialmente identificou-se os países que possuíam as línguas definidas no estudo entre suas línguas oficiais. Posteriormente realizou-se a busca das Bibliotecas destes países na plataforma Google Play, para levantamento dos aplicativos existentes. Tendo como resultado final da amostra para análise: 8 países e 11 aplicativos. Analisou-se as principais características presentes na utilização dos aplicativos e os serviços ofertados por eles. Dados os resultados obtidos, é possível concluir que as BN analisadas têm focado na oferta de serviços de difusão de informação e disponibilização de materiais, destacando-se a difusão de informações gerais das Bibliotecas e a oferta de e-books. Entre as conclusões, identificou-se uma baixa adoção das BN às tecnologias móveis. O foco parece estar nos serviços realizados presencialmente, como o depósito legal e a preservação das obras de seu país. Essa questão contraria a democratização do acesso à informação, um dos desafios da Sociedade da Informação.

Palavras-chave: Bibliotecas Nacionais. Serviços online. Aplicativos Móveis.

Bibliotecas nacionales y aplicaciones móviles: análisis de servicios en línea disponibles en aplicaciones para Android

Resumen: El objetivo de este estudio fue analizar los servicios en línea disponibles para aplicaciones de Android a partir de las bibliotecas nacionales (BN) de los países de la Información y el índice de desarrollo Tecnología de la Comunicación (IDI), que tiene entre sus idiomas oficiales son el español, Inglés o portugués, lo que resulta en total de 83 países. Inicialmente se identificaron los países que poseían las lenguas definidas en el estudio de sus lenguas oficiales. Posteriormente se realizó la búsqueda de las Bibliotecas de estos países en la plataforma Google Play, para levantar las aplicaciones existentes. Con el resultado final de la muestra para análisis: 8 países y 11 aplicaciones. Se analizaron las principales características presentes en la utilización de las aplicaciones y los servicios ofrecidos en los mismos. Dado que los resultados obtenidos, es posible afirmar que las BN analizadas se han centrado en la oferta de servicios de difusión de información y puesta a disposición de materiales, destacándose la difusión de informaciones generales de las Bibliotecas y la oferta de e-books. Entre las conclusiones, se identificó una baja adopción de las BN a las tecnologías móviles. El foco aún está en los servicios prestados 
presencialmente, como el depósito legal y la preservación de las obras de su país. Esta cuestión va en contra de la democratización del acceso a la información, un desafío de la Sociedad de la Información.

Palabras clave: Bibliotecas Nacionales. Servicios en línea. Aplicaciones Móviles.

National Libraries and mobile applications: analysis of online services available in Android applications Abstract: The aim of the study was to analyze the online services available in Android applications from the National Libraries (NL) of the ICT Development Index (IDI), which have Spanish, English or Portuguese as their official languages, resulting in a total of 83 countries. Initially, were searched the countries that had the languages defined in the study among their official languages. Later, the Libraries of this countries were searched on the Google Play platform for surveying existing applications. The result of the sample for analysis: 8 countries and 11 applications. We analyzed the main characteristics present in the use of the applications and the services offered in them. Given the results obtained, it is possible to affirm that the NL analyzed have focused on the provision of information dissemination services and material availability, especially the dissemination of general information on Libraries and the supply of eBooks. Among the conclusions, a low adaptation of NL to mobile technologies was identified. The focus is still on services performed on site, such as legal deposit and preservation of the works of his country. This contrary one of the challenges of the information society: the democratization of information access.

Keywords: National libraries. Online services. Mobile applications.

\section{Introdução}

O aumento na disponibilização de conteúdos na Internet e a facilidade ocasionada pelos dispositivos móveis alteram as formas de acesso e consumo de informação pelos indivíduos. Não há mais necessidade de compartilhar os mesmos tempo e espaço para haver interação entre pessoas, a busca e o acesso por informações pode ser feita com apenas alguns cliques, ou por comandos de voz. De acordo com o relatório The State of Broadband de setembro de 2017 da União Internacional de Telecomunicações (UIT), a agência de mídia Zenith estimou que 71\% de todo o consumo da Internet em 2016 ocorreu via celular e, em 2017, essas conexões somaram 7,7 bilhões, superando a população mundial, que totaliza aproximadamente 7,6 bilhões (INTERNATIONAL TELECOMMUNICATION UNION, 2017a).

Os computadores são muito utilizados pela população, porém os dispositivos móveis estão ganhando protagonismo com rapidez. Para ilustrar tal situação, veja-se que, em 2018, $52,2 \%$ de todo o acesso a internet foi gerado por meio de telefones celulares, em comparação a $50,3 \%$ no ano anterior (STATISTA, 2018).

A facilidade proporcionada pelos dispositivos móveis implica na exigência de que tudo ocorra de maneira mais simples, rápida e instantânea, influenciando também a forma de execução de diversas atividades humanas.

No contexto das Bibliotecas, o acesso remoto é indispensável, seja para realizar uma consulta ao catálogo, reservar ou renovar um livro, entre outros serviços. A utilização de sites e catálogos online pelas Bibliotecas supre apenas parcialmente essas necessidades, visto que, na atualidade, essas plataformas aparentemente são mais adequadas aos computadores do que aos dispositivos móveis. Uma biblioteca que se preocupa em estar presente nos ambientes 
próprios às tecnologias móveis, terá mais chances de manter seus usuários e atingirá potenciais usuários.

A fim de compreender como estão sendo ofertados os serviços online pelas Bibliotecas, é interessante mapear a sua atual situação perante a adoção das tecnologias móveis, em concreto, de aplicativos.

A presente pesquisa teve como principal objetivo analisar os serviços on-line disponíveis em aplicativos para Android de Bibliotecas Nacionais de países que possuem o espanhol, o inglês ou o português entre as suas línguas oficiais. Para o alcance do objetivo, foram realizadas as seguintes atividades: identificação dos aplicativos das Bibliotecas Nacionais dos países selecionados, na plataforma Google Play; categorização dos serviços disponibilizados nos aplicativos; análise das principais características dos aplicativos e dos serviços que ofertam.

\section{Sociedade da Informação}

Na era moderna, vivenciamos três grandes revoluções. A primeira foi há cinco séculos, quando Gutenberg, com sua imprensa, facilitou uma revolução nas informações e comunicações. Segundo Rayward (2014), essa revolução foi caracterizada pelo surgimento de infraestruturas de informação e comunicações institucionais complexas e interligadas e acabou sendo responsável por transformar as sociedades ocidentais. A segunda iniciou-se com o advento da Segunda Guerra Mundial, como consequência da tentativa de lidar com a crise na produção e gestão da informação, especialmente informações científicas e técnicas. Por sua vez, a terceira revolução iniciou-se na década de 1990 com o surgimento da World Wide Web (WWW) e dos sistemas e tecnologias de informação e comunicação (TIC) cada vez mais avançados. "Essa revolução tem como característica a transformação de nossa "cultura material" pelos mecanismos de um novo paradigma tecnológico que se organiza em torno da tecnologia da informação" (CASTELLS, 2016, p. 67).

As mudanças ocasionadas pelas revoluções tecnológicas afetam tanto aspectos sociais quanto econômicos, sejam as relações que possuímos, a maneira como nos comunicamos, a oferta de produtos e serviços ou o mercado de trabalho. Castells (2016) menciona que essas revoluções são caracterizadas por sua penetrabilidade em todos os domínios da atividade humana, e não como uma fonte externa de impacto.

A expressão "sociedade pós-industrial" era utilizada para descrever a sociedade à qual estávamos condicionados. Porém, por conta do novo paradigma tecnológico mencionado, tal expressão foi substituída por "Sociedade da Informação". Martin (1995 apud MUTULA, 2008) aponta que a Sociedade da Informação caracteriza-se pelo crescimento e uso da informação, e a exploração generalizada de diversas fontes de informação. É uma sociedade na qual os 
indivíduos possuem conhecimento das informações de que precisam; de onde e como obtê-las; e de que modo irão utilizá-las.

A Sociedade da Informação é impulsionada pelas TIC que, de acordo com Rao (2006, apud NWOSU; OGBOMO, 2010), estão se expandindo nos países em desenvolvimento. Entretanto, existe uma grande lacuna entre aqueles países que podem oferecê-las e os que não podem, resultando em indivíduos ainda desconexos desse paradigma tecnológico que estamos vivendo.

\section{TIC e tecnologias móveis}

É possível definir as TIC como "ferramentas utilizadas para coleta, processamento, armazenamento, transmissão e disseminação da informação." (EBIJUWA, 2005 apud ETEBU, 2010, p. 01). De acordo com Jaramillo e Patiño (2007), a sua evolução ocorreu de forma acelerada, alcançando uma compatibilidade surpreendente. Nesse sentido, os autores apontam três momentos importantes do desenvolvimento tecnológico: o primeiro orientado para o desenvolvimento de computadores; o segundo para o desenvolvimento da tecnologia como instrumento de armazenamento e distribuição; e o terceiro, mais recente, para a tecnologia de redes.

O mundo em rede gera e move grandes quantidades de informações eletrônicas, e caracteriza-se pelo acesso universal e de alta velocidade, e pela aplicação de informações e tecnologia de computadores em todos os âmbitos da sociedade (MUTULA, 2008). A American Library Association (2003) aponta que o mundo em rede vai além da Internet e conecta indivíduos a indivíduos (redes sociais), indivíduos a negócios, indivíduos à informação e indivíduos à cultura. Nesse contexto, os recursos digitais se tornam populares devido à sua facilidade de uso, mobilidade, compartilhamento de informações, acesso ilimitado e em qualquer lugar, baixo orçamento e investimento. (SAROJADEVI; PADMAMMA; WALMIKI, 2016).

A utilização de tecnologias móveis impacta em como as organizações ofertam seus produtos e serviços. Um site acessado por meio de um computador não será visualizado da mesma forma que em um tablet ou um dispositivo móvel, a menos que seja um site com design responsivo. De acordo com Bohyun (2013), o web design responsivo tem como objetivo fazer uma página da web parecer igualmente boa, independentemente do tamanho da tela do dispositivo.

Embora o design responsivo de sites possua grandes vantagens, isso por si só não garante uma experiência móvel satisfatória. Assim, além da adaptação do design de sites, algumas organizações investem na disponibilização de aplicativos móveis. Os aplicativos são softwares que precisam ser instalados nos dispositivos para utilização de seus recursos. De 
acordo com Ghuloum (2017), os aplicativos estão se tornando a maneira preferida ao acessar informações. Normalmente estão disponíveis e podem ser adquiridos em lojas de aplicativos operadas pelos proprietários do sistema operacional (SO), como Android ou iOS. Em março de 2017, havia 2,8 milhões de aplicativos disponíveis na Google Play Store e 2,2 milhões na App Store, as duas principais lojas de aplicativos do mundo (STATISTA, [2017?]).

Atualmente, alguns aplicativos móveis podem oferecer melhor experiência ao usuário quanto a recursos gráficos ou interface, além de melhor aproveitamento aos recursos nativos do celular, como Bluetooth, GPS e câmera, permitindo e facilitando diferentes usos e inovação na oferta de serviços, como acontece, por exemplo no setor dos videogames para telefone.

\section{Bibliotecas e tecnologias}

O consumo move a economia e com isso a forma como os produtos e serviços vêm sendo ofertados. Há uma constante preocupação das entidades em compreender e atender às necessidades e expectativas dos indivíduos. Com as Bibliotecas isso não ocorre de maneira diferente, os profissionais atuantes precisam ser estratégicos, conhecer seus usuários reais e potenciais, quais informações eles consomem, os suportes e meios de acesso utilizados, assim, podendo adaptar seus serviços e produtos para o contexto atual.

Ao longo de sua história, as Bibliotecas passaram por diversas mudanças por conta das tecnologias. Um dos maiores exemplos foi a passagem da utilização do catálogo impresso em fichas para o catálogo on-line, além da automatização de diversas outras atividades. Conforme apontado por Ansari (2017) no paradigma tecnológico atual, os bibliotecários passam a ter responsabilidades diferentes, devem atuar além das paredes da Biblioteca, pois os usuários podem estar em qualquer parte do mundo. O autor ainda aponta que, com a rápida obsolescência da tecnologia, os bibliotecários devem possuir habilidades específicas para lidar com as mudanças.

Porém, algumas entidades e profissionais são resistentes à mudanças. Permanecem focados nas técnicas e serviços tradicionais e não usufruem dos benefícios proporcionados pelas tecnologias que vêm surgindo. Essa visão, juntamente com a ausência de investimento dos órgãos ou setores superiores, resulta em Bibliotecas estacionadas no passado, que não evoluem nem inovam em seus serviços e produtos.

\section{Bibliotecas Nacionais}

Tradicionalmente, as Bibliotecas Nacionais (BN) possuem uma ligação com o processo de consolidação dos Estados Unidos e da Europa. Além de recolher, catalogar e armazenar livros em suas estantes tiveram importância simbólica e política, por sempre estarem associadas a 
estratégias de conhecimento, razões do Estado e visões do mundo. (BIBLIOTECA NACIONAL, 2004). Detêm responsabilidades especiais, geralmente definidas em lei. Essas responsabilidades variam de acordo com o país, mas incluem principalmente:

\begin{abstract}
coleta via depósito legal da publicação nacional (impressa e eletrônica) e sua catalogação e preservação; a prestação de serviços centrais (referência, bibliografia, preservação, empréstimo) aos usuários, tanto diretamente como através de outras bibliotecas e centros de informação; a preservação e promoção do patrimônio cultural nacional; aquisição de pelo menos uma coleção representativa de publicações estrangeiras; a promoção da política cultural nacional; e liderança em campanhas nacionais de alfabetização. (INTERNATIONAL FEDERATION OF LIBRARY ASSOCIATIONS AND INSTITUTIONS, 2018, tradução nossa)
\end{abstract}

Uma das atividades de maior importância e visibilidade nas BN é o depósito legal, prática consolidada há séculos em alguns países. Na França, foi criado em 1537. Em Portugal, existe desde 1805. No Brasil, o decreto no 1825, data de 1907. Esse decreto necessita de atualização, por causa do perfil editorial brasileiro contemporâneo e, também, pelos novos suportes que surgiram, como o livro eletrônico (BIBLIOTECA NACIONAL, 2004).

As Bibliotecas e Arquivos Nacionais não são entidades estagnadas, são afetadas por mudanças políticas, sociais e econômicas. As políticas e funções adotadas por essas instituições podem, por sua vez, influir no progresso ou na regressão de uma sociedade (ESKANDER, 2011). Dessa forma, as BN possuem uma relevante responsabilidade social perante o seu país, e os seus serviços devem ser planejados e executados entendendo adequadamente a conjuntura atual e as peculiaridades e as demandas de sua população, com o intuito de expandir o acesso e disseminação da informação, contribuindo assim de maneira significativa para o alcance do ideal da Sociedade da Informação.

\title{
6. Procedimentos metodológicos
}

A presente pesquisa é caracterizada, quanto aos procedimentos metodológicos, como comparativa pois "esse método procede pela investigação de indivíduos, classes, fenômenos ou fatos, com vistas a ressaltar as diferenças e similaridades entre eles." (GIL, 2008, p.16). Quanto à abordagem do problema é qualitativa, pois "[tende-se] a analisar [os] dados indutivamente" (MENEZES, 2009, p.16), como também quantitativa, pois busca "traduzir em números opiniões e informações para classificá-las e analisá-las" (MENEZES, 2009, p.16).

Os objetos analisados foram sites e aplicativos compatíveis com dispositivos móveis cujo sistema operacional é o SO Android, de BN de países presentes no Índice de Desenvolvimento de Tecnologia da Informação e Comunicação (IDI) e têm como línguas oficiais: espanhol, inglês ou português. 
A delimitação do SO se justifica pelo fato de o Android estar à frente, em termos de venda e uso. De acordo com dados da International Data Corporation (2018), aproximadamente $85 \%$ do volume mundial de smartphones utiliza o sistema Android. A delimitação do idioma justifica-se pelo fato de que as línguas espanhol, inglês e português estão dentre as 8 mais faladas do mundo, juntamente de chinês, hindi, árabe, bengali e russo, segundo dados do site Ethnologue ([2018?]). Estas não foram consideradas na delimitação, por dificuldades de tradução ocasionadas pelos símbolos de seu alfabeto.

Como critério inicial para a definição e seleção dos países das BN presentes nesse estudo, utilizou-se o Índice de Desenvolvimento de Tecnologia da Informação e Comunicação (IDI) da União Internacional de Telecomunicações (UIT). A UIT é a Agência do Sistema das Nações Unidas dedicada a temas relacionados às TICs. (ONU BRASIL, [201-]). O índice utilizado faz parte de um relatório chamado "Measuring the Information Society Report" do ano de 2017 que está em seu segundo volume. Este relatório apresenta uma análise do perfil dos países, baseada em três áreas principais: serviços móveis; serviços fixos; e política do governo. (INTERNATIONAL TELECOMMUNICATION UNION, 2017b). Optou-se pela utilização desse índice pela sua cobertura das TIC, considerando a presença de 176 países na análise, e pelas fontes de obtenção dos dados utilizadas. Além disso, uma das áreas de análise do relatório aborda exclusivamente serviços móveis, objeto de estudo deste trabalho.

Para a seleção dos países considerou-se os países mais bem posicionados no Índice com espanhol, inglês ou português como línguas oficiais, resultando no total de 83 países. Foi utilizado o site Ethnologue ${ }^{1}$ para consulta das línguas por país.

Sendo assim, os 83 países do Índice segundo esses critérios foram, respectivamente: Reino Unido, Nova Zelândia, Austrália, Estados Unidos, Singapura, Irlanda, Malta, Espanha, Canadá, Andorra, Bahrein, Barbados, São Cristóvão e Névis, Emirados Árabes Unidos, Uruguai, Portugal, Argentina, Brunei, Chile, Bahamas, Costa Rica, Malásia, Brasil, Trindade e Tobago, Maurício, Granada, Antígua e Barbuda, São Vicente e Granadinas, Colômbia, Maldivas, Venezuela, México, Seicheles, África do Sul, Cabo Verde, Panamá, Peru, Equador, Jamaica, Filipinas, Santa Lúcia, Botswana, República Dominicana, Fiji, Tonga, Bolívia, Paraguai, Gana, Sri Lanka, Namíbia, El Salvador, Belize, Butão, Timor-Leste, Guiana, Guatemala, Samoa, Honduras, Nicarágua, São Tomé e Príncipe, Lesoto, Índia, Zimbábue, Cuba, Quênia, Vanuatu, Nigéria, Gâmbia, Sudão, Zâmbia, Paquistão, Camarões, Moçambique, Uganda, Ruanda, Quiribati, Ilhas Salomão, Angola, Guiné Equatorial, Tanzânia, Malawi, Guiné-Bissau e Eritreia.

\footnotetext{
${ }^{1}$ https://www.ethnologue.com
} 
Primeiramente, realizou-se pesquisa dos sites das BN de cada país no buscador Google, para verificação do nome de cada uma delas. Posteriormente, realizou-se acesso aos sites por meio do smartphone a fim de verificar quais possuíam design responsivo. Por fim, realizou-se pesquisa dos nomes das Bibliotecas na plataforma Google Play ${ }^{2}$, a fim de identificar quais possuíam aplicativos para Android.

Realizou-se pesquisa no Google para identificar quais Bibliotecas da amostra possuíam sites. Com os sites mapeados, realizou-se acesso a três páginas de cada um destes, para verificar se seu design era responsivo. As páginas acessadas para verificação foram: página inicial (primeiro nível), catálogo (se houvesse) e outra página escolhida de maneira aleatória (segundo nível).

Posteriormente, realizou-se uma análise dos aplicativos, a fim de identificar os serviços ofertados. Para isso, primeiramente analisou-se as características dos aplicativos, considerando as que mais se destacavam, sendo elas: acesso por login e senha, necessidade de conexão persistente, opções de idiomas e atualização dos aplicativos

Em seguida, realizou-se o levantamento dos serviços ofertados e buscou-se agrupá-los em categorias que representassem o objetivo em comum dos diferentes grupos de serviços. As categorias definidas foram: difusão da informação; comunicação; acesso ao catálogo; e disponibilização de materiais.

$\mathrm{O}$ acesso aos sites e aplicativos foi realizado em um smartphone da marca Motorola, modelo Moto G5s, versão 7.1.1 do SO Android durante o período de julho a setembro do ano de 2018. A pesquisa e instalação dos aplicativos foi realizada por meio da plataforma Google Play.

Durante a utilização dos aplicativos, não foi possível obter acesso aos aplicativos BARD Mobile (Estados Unidos), Biblioteca Pública Digital (Chile) e BNP digital (Peru). A solução encontrada para suprir essa limitação foi analisar as imagens e descrições apresentadas pela plataforma Google Play, e os termos de serviço dos aplicativos, onde foi possível extrair algumas informações.

\section{Resultados e discussão}

Um dos dados que mais chamou a atenção foi que entre os 83 países do IDI que possuem como línguas oficiais, o espanhol, inglês ou português, apenas 61 BN $(73,4 \%)$ possuem sites,

\footnotetext{
${ }^{2}$ https://play.google.com/store
} 
sendo que somente 24 deles $(39,3 \%)$ possuem design responsivo em todas as páginas consultadas, de primeiro e segundo nível.

Quanto aos aplicativos para Android, identificou-se que apenas 8 BN $(9,6 \%)$ os possuem. O quadro 1 apresenta os países, seguidos de sua posição no IDI, e suas respectivas Bibliotecas e aplicativos.

Quadro 1 - Países selecionados, posições no Índice de Desenvolvimento de Tecnologia da Informação e Comunicação do relatório "Measuring the Information Society Report" (2017), e suas respectivas Bibliotecas e aplicativos.

\begin{tabular}{|c|c|c|c|}
\hline País & $\begin{array}{l}\text { Posição } \\
\text { IDI }\end{array}$ & Biblioteca & Aplicativo \\
\hline \multirow{3}{*}{ Estados Unidos } & \multirow{3}{*}{160} & \multirow{3}{*}{ Library of Congress } & BARD Mobile \\
\hline & & & National Book Festival \\
\hline & & & Aesop For Children \\
\hline Singapura & 180 & National Library Board & NLB Mobile \\
\hline \multirow[b]{2}{*}{ Espanha } & \multirow[b]{2}{*}{270} & \multirow[b]{2}{*}{ Biblioteca Nacional de España } & BNE 300 Años \\
\hline & & & Quijote interactivo \\
\hline Chile & 560 & Biblioteca Nacional de Chile & Biblioteca Pública Digital \\
\hline Costa Rica & 60 은 & $\begin{array}{c}\text { Biblioteca Nacional Miguel Obregón } \\
\text { Lizano }\end{array}$ & SINABI \\
\hline Malásia & 630 & National Library of Malaysia & PNM eReader \\
\hline Brasil & 660 & Biblioteca Nacional do Brasil & Biblioteca Nacional do Brasil \\
\hline Peru & 960 & Biblioteca Nacional del Perú & BNP digital \\
\hline
\end{tabular}

Fonte: Elaborado pelos autores (2018).

A seguir estão apresentadas as características e os serviços ofertados pelos aplicativos.

\subsection{Características dos aplicativos}

Durante a utilização dos aplicativos, identificaram-se quatro características presentes que influenciam significativamente no seu uso inicial. São elas: acesso por login e senha, necessidade de conexão persistente, opções de idiomas e atualização dos aplicativos

\subsubsection{Acesso por login e senha}

Entre os aplicativos analisados, 7 aplicativos solicitam acesso por login e senha, sendo que em 4 aplicativos o login é restrito a determinadas categorias.

O fornecimento de login e senha para acesso ao aplicativo BARD Mobile (Estados Unidos) é restrito a pessoas cegas, deficientes visuais ou deficientes físicos, que sejam residentes dos Estados Unidos e cidadãos dos Estados Unidos que vivem no exterior. $\mathrm{O}$ aplicativo Biblioteca Pública Digital (Chile) é restrito a chilenos, residentes estrangeiros no Chile e passageiros de voos 
nacionais. Já o BNP digital (Peru) é restrito a residentes e cidadãos do Peru. Só é permitido o cadastro com o DNI (registro de identificação nacional do Peru). No aplicativo NLB Mobile (Singapura) também há fornecimento de login e senha restrito, porém somente para a utilização de alguns serviços. Seu acesso é restrito a cidadãos ou residentes permanentes de Singapura.

Os aplicativos PNM eReader (Malásia), National Book Festival (Estados Unidos) e SINABI (Costa Rica) também solicitam login e senha para a utilização dos serviços, porém, diferente dos mencionados anteriormente, o acesso é liberado a qualquer indivíduo que possua interesse em utilizá-los.

\subsubsection{Necessidade de conexão persistente à Internet}

Esta categoria aborda a necessidade de acesso persistente à Internet, posteriormente ao download de cada aplicativo, isto é, durante a utilização dos serviços disponibilizados pela biblioteca.

Dos 11 aplicativos analisados, o Aesop For Children (EUA) é o único que não exige acesso persistente à Internet para utilização de todos os seus serviços. Após o download do acervo, a leitura das fábulas ofertadas no aplicativo, pode ser realizada no modo completamente off-line.

Entre os demais, 7 aplicativos requisitam acesso persistente, por login e senha, para o uso de qualquer serviço. Os 3 aplicativos restantes permitem um acesso misto: o catálogo da biblioteca e outros serviços, possuem acesso aberto e on-line; alguns outros conteúdos podem ser baixados, para leitura off-line. Por exemplo, no aplicativo BARD Mobile (Estados Unidos), é necessário ao acesso persistente, para que o usuário usufrua de alguns serviços, enquanto, no caso os audiobooks, é necessário fazer o download do conteúdo desejado.

O NLB Mobile, de Singapura, apresenta diversas informações que são atualizadas constantemente, sendo necessário o acesso persistente à Internet. Além disso, também permite que o usuário acesse sua conta da Biblioteca e realize ações como o empréstimo de materiais diretamente pelo aplicativo.

O serviço oferecido pelo aplicativo Biblioteca Nacional do Brasil é o acesso on-line ao catálogo da Biblioteca, o que também justifica a necessidade de conexão persistente.

\subsubsection{Opções de idiomas}

A barreira linguística pode ser um problema enfrentado pelos indivíduos. Em sua pesquisa, Shäffer (2007) comenta sobre aspectos responsáveis pela exclusão digital, que têm impedido sociedades de usufruírem das tecnologias da informação, da Internet, e de outros recursos. Entre esses aspectos está a linguagem, mais especificamente a ausência de conhecimento dos usuários perante outras línguas que não sejam as de seu país. A autora 
apresenta como sugestão para esse problema linguístico que afeta o ciberespaço, contínuos investimentos em tecnologias de tradução.

Pensando na diversidade linguística existente, resultante de sua história, imigração ou turismo, é recomendável que instituições como as BN se preocupem em oferecer recursos de idiomas em suas aplicações, visto que são guardiãs de acervos representativos no seu país,.

Nos aplicativos analisados, somente 2 apresentam opções de alteração de idioma. 0 aplicativo PNM eReader (Malásia) apresenta a opção de alteração do idioma, estando disponíveis os idiomas malaio e inglês. O aplicativo Biblioteca Nacional do Brasil (Brasil) permite a alteração para os idiomas catalão, inglês, espanhol, basco, galego e português.

\subsubsection{Atualização dos aplicativos}

Outra característica observada, foi a ausência de atualização em alguns aplicativos. Dos 11 aplicativos, apenas 6 foram atualizados no ano de 2018.

A atualização influencia na qualidade do aplicativo, quando não realizada regularmente, os recursos e funcionalidades se tornam obsoletos, muitas vezes o aplicativo pode passar a apresentar falhas no seu funcionamento. Os SO dos dispositivos móveis são constantemente atualizados, fazendo com que surjam novas versões, e dessa forma, caso os aplicativos não se adequem às mudanças, pode ocorrer de se tornarem incompatíveis com a versão atual dos SO. Além disso, as informações presentes no aplicativo precisam ser atualizadas, como por exemplo, a inserção de novos materiais e conteúdo.

O aplicativo Quijote interactivo (Espanha) apresentou erros, supõe-se que por conta da ausência de atualização, considerando que sua última atualização ocorreu no ano de 2015. Em sua utilização, ao clicar na opção de compartilhamento, o aplicativo encerra, não permitindo que o usuário continue as ações que estavam sendo realizadas. Além disso, ao clicar na opção de Menu, as informações que deveriam ser apresentadas não aparecem na tela.

\subsection{Serviços ofertados nos aplicativos}

Com a facilidade proporcionada pelo acesso à Internet, ampliam-se seus potenciais usuários e consequentemente os diferentes perfis e necessidades a serem atendidas. Por meio dos aplicativos móveis, as BN possuem oportunidade de expandir seu público, compartilhando o trabalho realizado referente à preservação do patrimônio cultural de seu país, disseminando sua coleção, e prestando serviços de maneira criativa e inovadora.

Durante a utilização dos aplicativos, verificou-se uma variedade nos serviços ofertados. Mesmo quando enquadrados com a mesma tipologia de serviço, a oferta em cada Biblioteca é realizada de maneira diferente, cada qual com suas características. Dessa forma, os serviços 
foram organizados em quatro categorias: difusão da informação, comunicação, acesso ao catálogo e disponibilização de materiais.

\subsubsection{Difusão da informação}

O serviço de difusão da informação consiste em transmitir ao usuário as informações das quais ele necessita. Nos aplicativos analisados, considerou-se como serviços de difusão da informação: serviços de notícias, agenda e informações gerais.

\section{Serviço de notícias}

Somente 1 aplicativo oferta o serviço de notícias, o SINABI (Costa Rica). O aplicativo fornece notícias atuais, históricas e curiosidades, não só referente à BN, mas do país, como um todo. O software também dispõe de um serviço de alerta, no qual quando alguma notícia é inserida ao aplicativo, o usuário recebe uma notificação em seu dispositivo. A leitura das notícias não é realizada no aplicativo, são apresentados links para o acesso, estando disponíveis o Portal (site) ou Facebook da BN.

\section{Agenda}

O serviço de agenda consiste na disponibilização de datas e horários de eventos realizados ou relacionados às BN. Apenas 3 aplicativos apresentam esse serviço.

O aplicativo NLB Mobile (Singapura) mostra informações do evento como: nome do evento, data, horários de início e término, local, além de permitir a busca de eventos. O serviço contempla os eventos realizados na $\mathrm{BN}$ e nas Bibliotecas que integram seu sistema. Ao selecionar o evento desejado, o usuário é direcionado a uma página para realizar sua inscrição, quando necessário.

No aplicativo SINABI (Costa Rica) são apresentadas informações como: nome do evento, data, horário de início, local e público alvo. Estão disponíveis apenas eventos realizados na BN.

Nos Estados Unidos, o aplicativo National Book Festival é diferente dos mencionados anteriormente, por ser utilizado para um evento anual realizado na BN. Seu serviço de agenda, bastante completo, abrange as atividades ofertadas e é possível utilizar diversos filtros: cronograma completo, cronograma por palcos, cronograma por autor, programações especiais, painéis, entrevistas, lançamentos de livros, vendas e autógrafos de livros, recomendados para crianças e jovens, atividades familiares, desfile dos estados, pavilhão da Library of Congress, exibições e atividades, e sessões marcadas. Ao selecionar a atividade desejada, é possível realizar anotações sobre ela, criar um lembrete, adicionar à sua agenda, marcar e compartilhar uma foto. O aplicativo também oferta o serviço de agenda individual, no qual o usuário pode gerenciar as atividades de que deseja participar. 


\section{Informações gerais}

O serviço de informações gerais é ofertado em 5 aplicativos. Tal serviço consiste na disponibilização de informações referente às Bibliotecas ou ao tema específico do aplicativo.

No aplicativo National Book Festival (Estados Unidos) são fornecidas informações do evento, como sua apresentação, autores presentes, patrocinadores, mapa do local, entrevistados, vendas e autógrafos de livros, acessibilidade do local, dicas de segurança e locais para alimentação.

Pela sua parte, o NLB Mobile (Singapura) fornece informações das Bibliotecas que integram seu sistema, como: endereço, distância, horário de funcionamento, contato, mapa de localização e instruções de deslocamento às Bibliotecas.

No caso do SINABI (Costa Rica), também são fornecidas informações das Bibliotecas que integram seu sistema, porém somente endereço, horário de funcionamento e contato.

O BNE 300 Años (Espanha) é um aplicativo desenhado para o evento de aniversário de 300 anos da Biblioteca Nacional da Espanha, portanto fornece informações como: data de realização, horários de funcionamento, mapa das exposições, instruções de deslocamento até o local, vídeo de apresentação e créditos aos responsáveis pelo evento.

No Brasil, a BN fornece informações dos diferentes setores da Biblioteca, como: localização no prédio, contato, link no site da BN e horário de funcionamento.

\subsubsection{Comunicação}

Determinou-se, como serviço de comunicação, a interação entre as Bibliotecas e seus usuários, seja no atendimento de uma demanda, prestação de um auxílio, retirada de dúvidas, proposta de melhoria, entre outros. No dia a dia de uma Biblioteca, esse serviço geralmente está associado a atendimentos presenciais, contato via telefone, ou troca de e-mails. No caso dos aplicativos analisados, considerou-se como serviços de comunicação: FAQs, feedback, recomendação de materiais e interação com outros usuários.

\section{FAQs}

Apenas dois aplicativos ofertam um serviço de FAQs, que consiste numa listagem de perguntas mais frequentes, o que facilita a retirada de dúvidas e proporciona autonomia aos usuários. O primeiro, o aplicativo National Book Festival (Estados Unidos) emprega FAQs para a auxiliar na utilização e funcionalidades do aplicativo, infraestrutura e programação de eventos; O segundo, o NLB Mobile (Singapura), aplica esse recurso para resolver dúvidas sobre os serviços ofertados, utilização e funcionalidades do aplicativo. 


\section{Feedback}

O serviço de feedback permite que as Bibliotecas mensurem a satisfação de seus usuários, enviem consultas e comentários ou possam identificar pontos fortes e fracos dos seus serviços e produtos. Embora possa ser um serviço interessante para as Bibliotecas terem um retorno dos seus usuários, apenas 1 aplicativo, o NLB Mobile (Singapura), apresenta a opção. Neste aplicativo o usuário se identifica inserindo seu e-mail e telefone e realiza um comentário sobre o aplicativo, os serviços ofertados ou sobre a Biblioteca.

\section{Recomendação de materiais}

Uma forma de as Bibliotecas identificarem as necessidades dos seus usuários é a partir do serviço de recomendação de materiais. Novamente, a BN Singapura, com seu aplicativo NLB Mobile, é a única que possibilita que seus usuários registrados façam sugestões de materiais.

\section{Interação com outros usuários}

O serviço de interação com outros usuários é ofertado por somente 1 aplicativo, o National Book Festival (Estados Unidos). Embora não seja um serviço comumente oferecido pelas Bibliotecas, é bastante interessante, pois permite que os usuários criem comunidades e compartilhem seus interesses e sugestões.

O National Book Festival (Estados Unidos) permite que seus usuários adicionem participantes do evento, enviem mensagens e realizem convites para as atividades da programação, basicamente como um networking. Além disso, o aplicativo apresenta um feed de atividades, no qual os usuários realizam postagens que podem ser curtidas e comentadas.

\subsubsection{Acesso ao catálogo}

A disponibilização de catálogos online pelas Bibliotecas é essencial no contexto atual. Alguns catálogos estão disponíveis apenas para acesso por desktops, outros, adaptados a sites responsivos. Porém, seria interessante que as Bibliotecas que possuem aplicativos, incluam esse serviço.

As opções disponíveis aos usuários durante o acesso ao catálogo são diferentes, de acordo com a Biblioteca. Algumas permitem apenas a consulta aos materiais disponíveis no acervo, outras, o empréstimo, reserva ou renovação. Nos aplicativos analisados, estão disponíveis os serviços de: consulta, empréstimo e reserva.

\section{Consulta}

O serviço de consulta ao catálogo é ofertado por 4 dos aplicativos analisados. O NLB Mobile, permite a consulta por busca simples e avançada, com os filtros: título, autor, assunto, língua e tipo de material. De forma similar, a Biblioteca Nacional do Brasil também tem busca 
simples e avançada, junto com os filtros: título, autor, assunto, tipo de material e setor da Biblioteca.

Por outro lado, o SINABI da Costa Rica oferece esse serviço, mas apenas para buscas simples.

Por último, a Biblioteca Pública Digital de Chile, não permitiu verificar como o serviço de consulta ao catálogo é ofertado, pois é obrigatório acessar o aplicativo com um login que é restrito a determinadas categorias.

\section{Empréstimo}

São três os aplicativos que oferecem o serviço de empréstimo. Desses, foi possível avaliar o NLB Mobile, que realiza empréstimo de ebooks e Audiobooks, com limite de empréstimo de 8 materiais e devolução automática, após 21 dias. Os aplicativos da Biblioteca Pública Digital (Chile) e da BNP Digital (Peru), embora disponibilizem o empréstimo, não puderam ser analisados, porque requisitam login obrigatoriamente.

\section{Reserva}

Apenas dois aplicativos permitem a reserva de materiais desde os seus aplicativos: o NLB Mobile (Singapura) e a Biblioteca Pública Digital (Chile).

\subsubsection{Disponibilização de materiais}

As BN possuem características distintas dos outros tipos de Bibliotecas quanto à disponibilização de seus materiais. Normalmente, esse serviço está relacionado à disponibilização física do acervo, permitindo o empréstimo domiciliar aos usuários. Pelo fato de os acervos das BN possuírem valor histórico, seus materiais nem sempre podem ser retirados da Biblioteca. O que geralmente ocorre é a consulta local, quando necessário.

Dessa forma, as BN podem utilizar-se do ambiente online para realizar a disponibilização de seus materiais digitais. Divulgando seu acervo, expandindo e proporcionando o acesso aos usuários, além de contribuir para a conservação e a preservação de sua coleção.

Os aplicativos analisados disponibilizam os seguintes tipos de materiais: audiobooks, fábulas, ebooks, periódicos, documentos históricos e áudios.

\section{Audiobooks}

O acesso a audiobooks é ofertado em apenas 2 aplicativos. No BARD Mobile (Estados Unidos), é necessário que o usuário realize o download para poder escutar cada item. No NLB Mobile (Singapura), o usuário pode escutar os audiobooks no modo online ou por download.

\section{Fábulas}


Um aplicativo possui como serviço a oferta de fábulas, o Aesop For Children (Estados Unidos). As fábulas apresentam sempre uma imagem sobre seu tema, sendo algumas interativas, isto é, podem emitir sons ou se alterar, quando o usuário clica sobre elas.

ebooks

A praticidade proporcionada pelos ebooks vem alterando a forma como alguns indivíduos realizam suas leituras. Os suportes informacionais estão em constante evolução e, dessa forma, é interessante que as Bibliotecas busquem se adaptar, para atender às necessidades e expectativas de seus usuários.

A oferta de ebooks compõem os serviços de cinco dos aplicativos analisados. Em três deles, o NLB Mobile (Singapura), o BNP Digital (Peru) e o SINABI (Costa Rica), a leitura dos ebooks pode ser realizada de modo online ou por download.

O aplicativo PNM eReader (Malásia), conforme enuncia seu nome, funciona como um leitor eletrônico. Com seu login, o usuário pode realizar o gerenciamento dos eBooks baixados na Biblioteca, buscá-los por título, autor, e assunto, além de criar coleções com os títulos desejados.

Pelo fato de possuir acesso por login restrito a determinadas categorias, não foi possível obter informações suficientes em relação às funcionalidades de leitura de $e B o o k s$ no aplicativo Biblioteca Pública Digital (Chile).

\section{Periódicos}

A oferta de periódicos é um serviço de apenas 2 aplicativos analisados. Aqueles que aparecerem no aplicativo SINABI (Costa Rica) são revistas e jornais antigos. Ao acessá-los, o usuário é direcionado ao portal da $\mathrm{BN}$, onde pode selecionar o material por número e tomo, em caso de revistas, e dia, mês e ano de publicação, para jornais.

Não foi possível avaliar esta funcionalidade no caso do aplicativo NLB Mobile (Singapura), uma vez que o login é requerido, obrigatoriamente.

\section{Documentos históricos}

Apenas 2 aplicativos oferecem esse serviço, sendo os dois da Espanha. O BNE 300 Años, com documentos históricos, parte das exposições que comemoram os três séculos de história da entidade. Entre os documentos presentes no aplicativo estão: pinturas, livros, mapas, cantigas, documentos, partituras, diários, cartas e fotografias.

O aplicativo Quijote interactivo fornece documentos históricos referentes ao livro Dom Quixote de la Mancha, de Miguel de Cervantes. Entre os documentos, estão as edições de Dom Quijote organizadas por século e ano, além de mapas e imagens relacionados com a obra. 


\section{Áudios}

Um aplicativo possui, entre os seus serviços, a oferta de áudios não enquadrados como audiobooks. Trata-se do SINABI (Costa Rica), que apresenta uma Biblioteca de músicas. Essa biblioteca permite selecionar a música por região do país ou por tipo, sendo eles: acadêmica, popular, hinos e marchas, bandas, tradicionais e infantis. Além disso, também disponibiliza áudios históricos como anúncios comerciais, pronunciamentos e programas temáticos transmitidos por rádio.

\section{Considerações finais}

Destaca-se que entre os 83 países da amostra, apenas 73,4\% das BN (61 bibliotecas) possuem site, sendo que somente $26,2 \%$ (16 sites) têm um design responsivo. Se focarmos em quantos têm aplicativos para Android, uma cifra muito baixa, apenas 9,6\% (8 países), disponibiliza algum aplicativo no SO mais utilizado no mundo.

A restrição de acesso por obrigatoriedade de uso de login foi uma limitação significativa para esta pesquisa. Aplicativos como o BARD Mobile (Estados Unidos), o Biblioteca Pública Digital (Chile) e o BNP digital (Peru), limitam, de fato, a utilização dos aplicativos àqueles usuários que não são registrados, prejudicando assim o acesso ao serviço a usuários de outras partes do mundo. Além disso, verificou-se que outros aplicativos, embora permitam o acesso a determinados serviços, restringem seus usuários para algumas funcionalidades.

Referente à oferta de idiomas, é curioso observar o caso do aplicativo Biblioteca Nacional do Brasil. Dos 6 idiomas oferecidos, 4 são falados na Espanha (catalão, espanhol, basco e galego). Acredita-se que seria mais interessante focar a população brasileira, considerando que existem mais de 150 línguas indígenas no país, sendo que 5 delas contam com mais de 10 mil falantes, segundo o Censo demográfico do Instituto Brasileiro de Geografia e Estatística (2010).

Quanto à baixa oferta de serviços acessíveis, somente 2 dos aplicativos analisados ofertam audiobooks. Seria recomendável oferecer serviços adaptados a todos os usuários, incluindo aqueles com necessidades especiais, sem esquecer suas particularidades e possíveis restrições de uso ou acesso. Uma melhora desses serviços pode auxiliar na democratização do acesso à informação, um dos desafios da Sociedade da Informação.

Parece evidente, para o contexto avaliado, que as BN ainda estão muito focadas em serviços presenciais, como o depósito legal e a preservação das obras de seu país. É fato que os serviços presenciais e de preservação estão diretamente ligados à missão institucional das BN. Entretanto, a forma como os indivíduos acessam e consomem as informações está em constante mudança. Assim, as BN deveriam adaptar seus serviços, a fim prestarem uma contribuição 
relevante para a educação e cultura dos países, de modo a atender as necessidade dos seus usuários.

Considerando essa questão, a oferta de eBooks merece especial destaque. Apenas 5 aplicativos oferecem livros digitais, demonstrando que há alguma tentativa de adaptação aos novos suportes de leitura por parte das BN analisadas e expansão do acesso. Isso permitirá que usuários de qualquer parte do mundo desfrutem de uma parte do seu patrimônio cultural.

Em geral, observa-se a baixa oferta de serviços e quase a metade dos aplicativos já está desatualizada. Como se revela na pesquisa, a adesão da tecnologia móvel é tímida. Um cenário ideal seria que todas as BN possuíssem sites responsivos, com uma boa oferta de serviços online e as mínimas restrições, para que a maior quantidade de usuários nacionais ou estrangeiros pudesse desfrutar dos seus acervos e serviços. Seria desejável, também, a oferta de aplicativos com serviços inovadores e que aproximasse as entidades da população e de outras entidades.

Diversos fatores impactam no alcance desse ideal como, por exemplo, a economia. Os países vivem contextos e situações econômicas diferentes, tornando difícil o repasse de investimentos a determinados setores. Especialmente nos países mais pobres, outras questões acabam se tornando prioridade e subjugando as necessidades institucionais das BN. Como alternativa, os representantes das BN poderiam se associar e compartilhar projetos internacionais, a fim de demonstrar a importância do seu papel para a história dos países e para os cidadãos. Isso permitiria o compartilhamento de suas experiências e expertises, para a criação de um cenário que favoreça o cumprimento de sua missão na sociedade.

\section{Referências}

AMERICAN LIBRARY ASSOCIATION. Principles for the Networked World. 2003. Disponível em: $<$ http://www.ala.org/aboutala/sites/ala.org.aboutala/files/content/oitp/publications/issuebrie fs/principles/principles.pdf>. Acesso em: 19 out. 2018.

ANSARI, Munira Nasreen. Librarian as cybrarian. Pakistan Library \& Information Science Journal, Lahore, v. 38, n. 2, p. 24-31, jun. 2017. Disponível em: <http://web.b-ebscohostcom.ez46. periodicos.capes.gov.br/ehost/detail/detail?vid=2\&sid=9b8e2cc3-943f-4d70-adcea804cd7e81cc@sessionmgr104\&bdata=Jmxhbmc9cHQtYnImc2l0ZT1laG9zdC1saXZl\#AN=25497 $\underline{843 \& d b=l i h>}$. Acesso em: 1 jun. 2018.

BIBLIOTECA NACIONAL (Brasil). Biblioteca Nacional. São Paulo: Banco Safra, 2004. 351p.

CASTELLS, Manuel. A sociedade em rede. 17. ed. São Paulo: Paz e Terra, 2016. 698 p. (A era da informação ; economia, sociedade e cultura Vol. 1). ISBN 9788577530366.

ESKANDER, Saad B.. Iraq National Library and Archive: Inherited Difficulties and New Challenges. Alexandria, [S. I.], v. 22, n. 1, p. 47-51, Jan. 2011. Disponível em: <http://web.a-ebscohost- 
com.ez46. periodicos.capes.gov.br/ehost/detail/detail?vid=3\&sid=ef3a5199-029d-4863-8d007c877e861838@sessionmgr4006\&bdata=Jmxhbmc9cHQtYnImc2lOZT1laG9zdC1saXZl\#AN=703 50940\&db=lih>. Acesso em: 1 jun. 2018.

ETEBU, Abraham Tabor. ICT Availability in Niger Delta University Libraries. Library Philosophy \& Practice. [S .I.], p. 1-6, October 2010. Disponível em: <http://web.b-ebscohostcom.ez46. periodicos.capes.gov.br/ehost/detail/detail?vid=4\&sid=f58ca460-b414-45c9-99a7b7fbfdd36790@pdc-vsessmgr01\&bdata $=$ Jmxhbmc9cHQtYnImc2l0ZT1laG9zdC1saXZl\#AN=56660094\&db=lih $>$. Acesso em: 30 maio 2018.

ETHNOLOGUE. Summary by language size. [2018?]. Disponível em: <https://www.ethnologue.com/statistics/size>. Acesso em: 6 jun. 2019.

GIL, Antonio Carlos. Métodos e técnicas de pesquisa social. 6. ed. São Paulo: Atlas, 2008. 200 p.

GHULOUM, Husain F.; AL-LAMKI, Zuwainah. The utilization of smartphones apps as a service tool at Kuwaiti academic libraries. Informing Science, [S. I.], v. 20, n. 1, p. 133-148, June 2017. Disponível em: <http://web.a-ebscohostcom.ez46.periodicos.capes.gov.br/ehost/detail/detail?vid=2\&sid=074a04c5-8ed7-413f-9a93d7b6158094e6@sessionmgr4008\&bdata=Jmxhbmc9cHQtYnImc2l0ZT1laG9zdC1saXZI\#AN=124 138703\&db=lih>. Acesso em: 31 maio 2018.

INTERNATIONAL DATA CORPORATION. Worldwide smartphone volumes will remain down in 2018 before returning to growth in 2019 and beyond, according to IDC. Massachusetts: IDC, 2018. Disponível em: <https://www.idc.com/getdoc.jsp?containerld=prUS43856818>. Acesso em: 27 ago. 2018.

INTERNATIONAL FEDERATION OF LIBRARY ASSOCIATIONS AND INSTITUTIONS. National Libraries Section. 2018. Disponível em: <https://www.ifla.org/national-libraries/>. Acesso em: 9 jun. 2018.

INTERNATIONAL TELECOMMUNICATION UNION. The State of Broadband 2017: Broadband Catalyzing Sustainable Development. 2017a. Disponível em: $<$ https://www.itu.int/dms pub/itus/opb/pol/S-POL-BROADBAND.18-2017-PDF-E.pdf/>. Acesso em: 9 jun. 2018.

INTERNATIONAL TELECOMMUNICATION UNION. Measuring the Information Society Report 2017. 2017b. Disponível em: <https://www.itu.int/en/ITUD/Statistics/Documents/publications/misr2017/MISR2017 Volume1.pdf>. Acesso em: 5 maio 2018.

INSTITUTO BRASILEIRO DE GEOGRAFIA E ESTATÍSTICA. Censo demográfico 2010: características gerais dos indígenas: resultados do universo. 2010. Disponível em: $<$ https://biblioteca.ibge.gov.br/pt/biblioteca-catalogo?view=detalhes\&id=795 >. Acesso em: 20 out. 2018.

JARAMILLO, Orlanda; PATIÑO, José Daniel Moncada. La biblioteca pública y las Tecnologías de la Información y las Comunicaciones (TIC): una relación necesaria. Revista Interamericana de Bibliotecologia, Medellín, v. 30, n. 1, p. 15-50, jun. 2007. Disponível em: <http://web.bebscohost-com.ez46. periodicos.capes.gov.br/ehost/detail/detail?vid=2\&sid=f58ca460-b41445c9-99a7-b7fbfdd36790@pdc-vsessmgr01\&bdata $=$ Jmxhbmc9cHQtYnImc2l0ZT1laG9zdC1saXZ|\#AN=27047067\&db=lih>. Acesso 
em: 2 jun. 2018.

KIM, Bohyun. Responsive Web Design, Discoverability, and Mobile Challenge. Library Technology Reports, Chicago, v. 49, n. 6, p. 29-38, Sept. 2013. Disponível em: <http://web-bebscohost.ez46. periodicos.capes.gov.br/ehost/detail/detail?vid=2\&sid=395af3c9-f6bd-40008584c47e3b5bf621\%40sessionmgr104\&bdata=Jmxhbmc9cHQtYnImc2I0ZT1laG9zdC1saXZl\#AN=904 05356\&db=lih>. Acesso em: 10 abr. 2019.

MENEZES, Estera Muszkat. Pesquisa bibliográfica. Florianópolis: CIN; CED; UFSC, 2009. 86 p.

MUTULA, Stephen M.. Information and Computer Technology Management Challenges in the Networked World: Implications for Libraries in Africa. Journal Of Interlibrary Loan, document Delivery \& Electronic Reserve, [S. I.], v. 19, n. 1, p. 57-73, Jan. 2009. Disponível em: $<$ https://searchproquest.ez46.periodicos.capes.gov.br/lisa/docview/57683193/A959049D04334AFCPO/1?acc ountid=26642>. Acesso em: 31 maio 2018.

NWOSU, Obiora; OGBOMO, Esoswo F. The Impact of the Information Society on the Library and Information Science Profession. Library Philosophy \& Practice. [S. I.], p. 1-3, Oct. 2010. Disponível em: <http://web.b-ebscohostcom.ez46. periodicos.capes.gov.br/ehost/detail/detail?vid=2\&sid=43a6dc78-2c93-4350-a6ca7083aecfb140@sessionmgr104\&bdata=Jmxhbmc9cHQtYnImc2l0ZT1laG9zdC1saXZI\#AN=56660 $131 \& \mathrm{db}=$ lih>. Acesso em: 31 maio 2018.

ONU BRASIL. Nações Unidas. UIT: União Internacional de Telecomunicações. [201-]. Disponível em: <https://nacoesunidas.org/agencia/uit/>. Acesso em: 5 maio 2018.

RAYWARD, W. Boyd. Information Revolutions, the Information Society, and the Future of the History of Information Science. Library Trends, [S .I.], v. 62, n. 3, p. 681-713, Sept. 2014. Disponível em: <http://web.b-ebscohostcom.ez46.periodicos.capes.gov.br/ehost/detail/detail?vid=2\&sid=e3111e36-056f-42ed-a6efbf2c7fcd2945@sessionmgr101\&bdata=Jmxhbmc9cHQtYnImc2I0ZT1laG9zdC1saXZl\#AN=95782 $\underline{075 \& d b=l i h>}$. Acesso em: 31 maio 2018.

SAROJADEVI, K.; PADMAMMA, S.; WALMIKI, R.H. Digital Resource Management Strategies: Study on Selected All India Radio Media Libraries of Karnataka. Desidoc Journal Of Library \& Information Technology, [S .I.], v. 36, n. 6, p. 390-395, Nov. 2016. Disponível em: $<$ https://search-

proquest.ez46.periodicos.capes.gov.br/lisa/docview/1856861626/5EED4360090A4EB3PQ/1?a ccountid=26642>. Acesso em: 30 maio 2018.

SHÄFFER, Ana Maria de Moura. As novas tecnologias e a In(ex)clusão social, linguística e digital. Acta Científica, São Paulo, v. 1, n. 12, p. 61-70, jan. 2007. Semestral. Disponível em: <https://revistas.unasp.edu.br/acch/article/view/460>. Acesso em: 15 set. 2018.

STATISTA. Mobile App Usage: Statistics \& Facts. [2017?]. Disponível em: <https://www.statista.com/topics/1002/mobile-app-usage/>. Acesso em: 9 jun. 2018.

STATISTA. Percentage of all global web pages served to mobile phones from 2009 to 2018. 2018. Disponível em: <https://www.statista.com/statistics/241462/global-mobile-phonewebsite-traffic-share/>. Acesso em: 6 jun. 2019. 
